\title{
Desarrollo en Blender del gemelo digital de una estación electro-neumática para su aplicación en laboratorios virtuales
}

\section{Development in Blender of the digital twin of an electro-pneumatic station for its application in virtual laboratories}

CARMONA-MARTÍNEZ, Luis Alberto†**, GÓMEZ-HERNÁNDEZ, Alejandro, ORTÍZ-ORTÍZ, Tania Judith y GUALITO-OLVERA, Miguel Ángel

\section{Universidad Tecnológica de Querétaro}

ID 1er Autor: Luis Alberto, Carmona-Martínez / ORC ID: 0000-0002-7925-1229, CVU CONACYT ID:486128

ID 1er Coautor: Alejandro, Gómez-Hernández / ORC ID: 0000-0003-2089-9829, CVU CONACYT ID: 486382

ID 2do Coautor: Tania Judith, Ortíz-Ortíz / ORC ID: 0000-0001-6712-9237, CVU CONACYT ID: 426398

ID 3er Coautor: Miguel Ángel, Gualito-Olvera / ORC ID: 0000-0001-5138-0961, CVU CONACYT ID: 864375

DOI: $10.35429 / J T E N .2019 .11 .3 .1 .7$

Recibido 03 de Julio, 2019; Aceptado 09 Septiembre, 2019

\section{Resumen}

Este artículo presenta el desarrollo del gemelo digital de una estación electro-neumática compuesta con 3 pistones, para implementar esta estación en laboratorios virtuales. El objetivo de esta estación es contar con una alternativa económica para la realización de prácticas de automatización que cuente con la capacidad de conectarse a través de Ethernet con PLCs de la marca de Allen Bradley y con todos los PLCs que se comuniquen bajo el protocolo Modbus TCP. Para desarrollar el gemelo digital presentado en este artículo se tomó como referencia una estación física electro-neumática del laboratorio de Mecatrónica en la universidad tecnología de Querétaro, el desarrollo este gemelo digital se hizo a través del software gratuito Blender complementándolo con programación en Python para la comunicación Ethernet TCP con los distintos controladores lógicos programables. Posteriormente se realizó la validación de este proyecto mediante la conexión tanto del gemelo digital como de la estación física tomada como referencia, con un PLC ControlLogix. Este trabajo permitirá a futuro implementar en universidades, centros de capacitación e instituciones interesadas, laboratorios virtuales de bajo costo, con múltiples escenarios de prácticas, que den como resultado estudiantes mejor preparados en la programación de sistemas automatizados en la industria.

Gemelos digitales, Laboratorios virtuales, Blender

\begin{abstract}
This paper presents the development of the digital twin of an electro-pneumatic station composed with 3 pistons, to implement this station in virtual laboratories. The objective of this station is to have an economical alternative for the accomplishment of practices of automation that counts on the capacity to connect through Ethernet with PLCs of the mark of Allen Bradley and with all the PLCs that communicate under the protocol Modbus TCP. Later, in order to develop the digital twin presented in this paper, an electro-pneumatic physical station was taken from the Mechatronics laboratory at the Universidad Tecnológica de Queretaro, the development of this digital twin was done through the free software Blender, complementing it with programming in Python for the Ethernet TCP communication with the different programmable logic controllers. Finally, we carried out the validation of this project through the connection of both the digital twin and the physical station taken as a model, with a ControlLogix PLC. This work will allow the future to implement low-cost virtual laboratories in universities, training centers and interested institutions. The laboratories will have multiple practice scenarios that result in students better prepared in the programming of automated systems in the industry.
\end{abstract}

Digital twins, Virtual laboratories, Blender

Citación: CARMONA-MARTÍNEZ, Luis Alberto, GÓMEZ-HERNÁNDEZ, Alejandro, ORTÍZ-ORTÍZ, Tania Judith y GUALITO-OLVERA, Miguel Ángel. Desarrollo en Blender del gemelo digital de una estación electro-neumática para su aplicación en laboratorios virtuales. Revista de Ingeniería Tecnológica. 2019 3-11: 1-7

\footnotetext{
* Correspondencia del Autor (Correo electrónico: alberto0488@ hotmail.com)

$\uparrow$ Investigador contribuyendo como primer autor.
} 


\section{Introducción}

La competitividad altamente creciente en la industria de hoy exige productos con calidad y precios competitivos.

Para superar este desafío muchas industrias han integrado sistemas automatizados, reemplazando la toma de decisiones del ser humano, en un proceso mecanizado, por computadoras y dispositivos de automatización, dejando el control de las líneas de producción a una cantidad mínima de operadores.

La automatización hoy en día es la implementación de robots y sistemas computacionales que se apoyan de la inteligencia artificial y el big data para realizar las actividades rutinarias con una mejor calidad y a menor costo. Se considera que el mercado de la automatización llegue a 149 mil millones de dólares para el año 2022, significando un crecimiento promedio anual de un 6\% (Market Research Future, 2018).

Otro factor muy importante a considerar es la transición a la cuarta revolución industrial o mejor conocida como industria 4.0, teniendo como objetivo la puesta en marcha de un gran número de fábricas inteligentes, este concepto implica la completa digitalización de las cadenas de valor, teniendo como resultado un alto grado de automatización y digitalización de las fábricas.

México ocupa un lugar privilegiado entre los países con más egresados de carreras orientadas a la ciencia, tecnología, ingeniería y matemáticas, conocidas como carreras STEM por sus siglas en inglés (Science, Technology, Engineering and Mathematics).

La Organización para la Cooperación y el Desarrollo Económico (OCDE) señala a México con una de las mayores proporciones de estudiantes que ingresan a carreras STEM, con un $35 \%$, ocupando el cuarto lugar en número de egresados de dichas carreras, quedando apenas por debajo de Alemania, Estonia y Finlandia. En contraste con el lugar ocupado en carreras STEM, en México la educación técnica solo representa el $4 \%$ de la educación total, mientras que en Alemania representa casi el $45 \%$ (Forbes México, 2018).
Para que México tenga profesionales que cumplan las competencias y requerimientos en automatización industrial es necesario un constante entrenamiento, el cual inicia desde las instituciones educativas en los laboratorios y continúa en los centros de entrenamiento personal para la automatización. Desafortunadamente, muchos de estos laboratorios cuentan con infraestructura limitada, especialmente en las universidades públicas.

Dentro de los temas más importantes para el entrenamiento en automatización en ambiente industrial resaltan los siguientes:

\section{- $\quad$ Programación de PLCs \\ - Robótica \\ - Control de Movimiento \\ - Control Automático \\ - Protocolos de comunicación industrial}

Tomando en cuenta los temas y la necesidad de calidad en el entrenamiento de automatización, el cuerpo académico de automatización y control, de la Universidad Tecnológica de Querétaro, propone una alternativa educativa de bajo costo, que permita a los estudiantes poder interactuar de manera virtual con escenarios industriales bajo el concepto de gemelo digital, ofreciendo los mismos retos en programación, robótica, protocolos de comunicación y control de movimiento.

Estos escenarios solo representaran la parte mecánica, de sensores y actuadores del proceso y para ellos deberán contar con un complemento físico, como lo puede ser un Controlador Lógico Programable, permitiendo al estudiante programar equipos industriales comerciales aplicados en escenarios virtuales. Debido al alto costo de los equipos comerciales aplicados en la industria, en este artículo también se implementa una opción física educativa de bajo costo para ser implementado como controlador lógico programable en estos escenarios. Para el desarrollo de este primer escenario virtual se realizó un estudio de varios artículos relacionados con laboratorios virtuales.

Uno de ellos se centra en el desarrollo de un laboratorio virtual creado con el software gratuito Phys $\mathrm{X}^{\circledR}$ (Ortega et. al., 2017), el cual consiste en un escenario simulado compuesto por sensores, pistones, motores y un robot delta.

CARMONA-MARTÍNEZ, Luis Alberto, GÓMEZ-HERNÁNDEZ, Alejandro, ORTÍZ-ORTÍZ, Tania Judith y GUALITO-OLVERA, Miguel Ángel. Desarrollo en Blender del gemelo digital de una estación electroneumática para su aplicación en laboratorios virtuales. Revista de Ingeniería Tecnológica. 2019 
Todo el sistema cuenta con comunicación con controladores lógicos programables a través del protocolo Modbus TCP.

Un segundo trabajo encontrado consiste en el desarrollo de un laboratorio virtual, que parte de una iniciativa del Sistema Tecnológico de Monterrey, con el objetivo de mejorar, apoyar y desarrollar una nueva infraestructura de laboratorios para el nuevo modelo educativo del siglo XXI. El proyecto fue implementado con el software comercial Labview a través de modelos 3D bajo el formato VRML (Virtual Reality Modeling Languaje) para ser ensamblados y convertido en un escenario para el laboratorio virtual, dicho laboratorio cuenta con comunicación PROFINET para controladores lógicos programables de la marca Siemens (Antonio et. al., 2011).

Un tercer trabajo encontrado consiste en la implementación de un laboratorio virtual para la enseñanza de controladores PDI (Márquez et. al., 2008), este laboratorio no cuenta con conexión a algún equipo industrial externo y su desarrollo fue principalmente con el software Matlab.

El valor agregado respecto a los trabajos encontrados consiste en la comunicación con controladores lógicos programables de la marca de Allen Bradley ${ }^{\circledR}$ y con equipos que se comuniquen con Modbus TCP.

La estructura del primer escenario para el laboratorio virtual se basa en un gemelo digital desarrollado con el software gratuito Blender ${ }^{\circledR}$ de una estación electro neumática del laboratorio de la Universidad Tecnológica de Querétaro (Figura 1).

Este escenario cuenta con scripts en lenguaje Python, desarrollados en este trabajo, que le permiten comunicarse con Ethernet industrial. Este protocolo de comunicación se eligió debido al rápido crecimiento que tiene en el mercado. Con una tasa de crecimiento de un $20 \%$, representando en el 2016 el $38 \%$ del mercado global (Hardware Meets Software, 2018). Es necesario mencionar que el Ethernet industrial es la aplicación del estándar Ethernet en un entorno industrial con diversos protocolos que proporcionan determinismo y control.

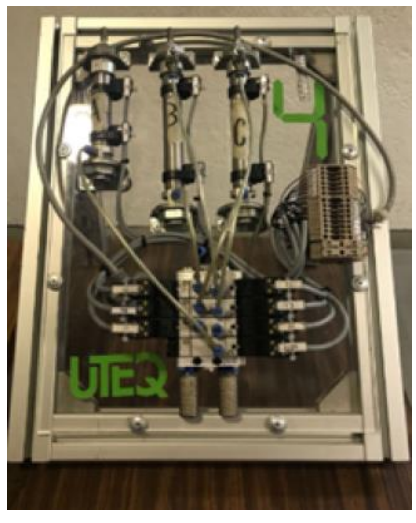

Figura 1 Estación electroneumática Fuente: Elaboración Propia

Dentro de la diversidad del protocolo del Ethernet industrial, se eligieron dos protocolos para este primer avance. El primero es el protocolo EtherNet/IP, el cual ocupa el primer lugar de los protocolos del Ethernet industrial en el mercado (Hardware Meets Software, 2018).

El protocolo EtherNet/IP también es conocido como "ControlNet sobre Ethernet" y consiste en la aplicación del protocolo CIP en la capa de transporte, sesión, presentación y aplicación del modelo de referencia OSI. Utilizando como capa física y capa de enlace de datos el estándar Ethernet y respectivamente IP, UDP/TCP; como la capa de red y de transporte.

El segundo es el protocolo de comunicación Modbus TCP, este protocolo ocupa el cuarto lugar de los protocolos del Ethernet industrial en el mercado, pero fue elegido debido a que distintas marcas de PLC lo manejan. Dándole así a este proyecto una gran diversidad de equipos con los cuales puede comunicarse, y permitiendo también en este artículo agregar como complemento de controlador lógico programable una Raspberry Pi 3 programada con el software industrial de bajo costo Codesys ${ }^{\circledR}$

El artículo está estructurado de la siguiente manera: En la sección 2 se presenta la metodología utilizada para el proyecto. En la sección 3 se presenta la arquitectura del ambiente virtual y de los protocolos de comunicación.

En la sección 4 se muestra el impacto del laboratorio virtual dentro de las carreras en la universidad tecnológica de Querétaro. Finalmente en la sección 5 se presentan las conclusiones del trabajo. 


\section{Metodología}

La metodología implementada para desarrollar el proyecto se muestra en la figura 2 .

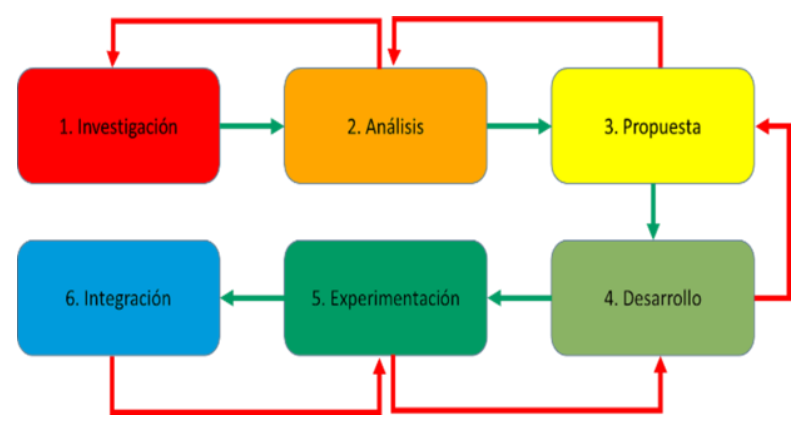

Figura 2 Diagrama de la metodología Fuente: Elaboración Propia

1. Investigación del estado del arte respecto a los proyectos y aplicaciones similares.

2. Análisis de los softwares disponibles para el desarrollo de ambientes virtuales y los requerimientos técnicos para poder llevar a cabo el proyecto.

3. Propuesta del software a utilizar, escenario a diseñar y protocolos de comunicación.

4. Desarrollo del ambiente virtual y programación de los protocolos industriales dentro del mismo.

5. Integración e implementación del escenario virtual con un controlador lógico programable.

\section{Arquitectura}

A continuación se presenta el desarrollo de del trabajo realizado, primero se da a conocer el diseño y la arquitectura dentro de Blender y luego la arquitectura de comunicación de los protocolos industriales implementados en este trabajo.

\section{Diseño y arquitectura en Blender}

Blender es un programa que integra una serie de herramientas para la creación de contenido 3D destinado a realización de imágenes estáticas, videos de alta calidad y creación de contenido interactivo, Blender es un "software libre", con el código fuente disponible bajo la licencia GNU GPL.

Para el desarrollo del ambiente virtual se realizó en SolidWorks un modelo en 3D de una de las estaciones electroneumáticas del laboratorio de la universidad tecnológica de Querétaro (Figura 3).

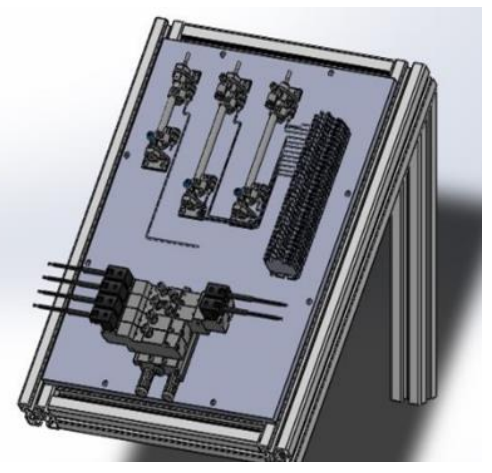

Figura 3 Estación electroneumática en SolidWorks Fuente: Elaboración Propia

Los modelos en 3D son exportados en formato stl, lo que permite a Blender leer, abrir y visualizar el ensamble de la estación (Figura 4).

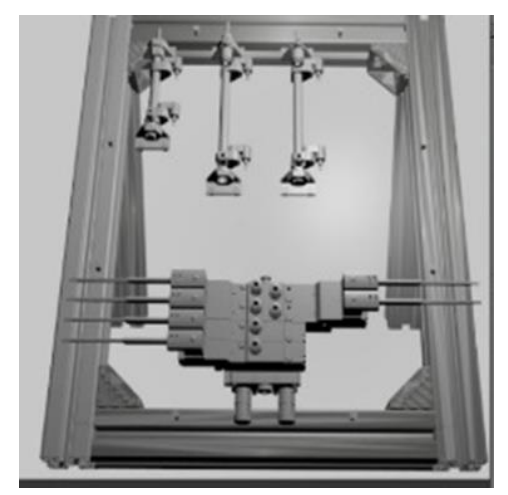

Figura 4 Estación electroneumática en Blender Fuente: Elaboración Propia

Para el desarrollo del ambiente virtual se partió del siguiente diagrama (Figura 5).

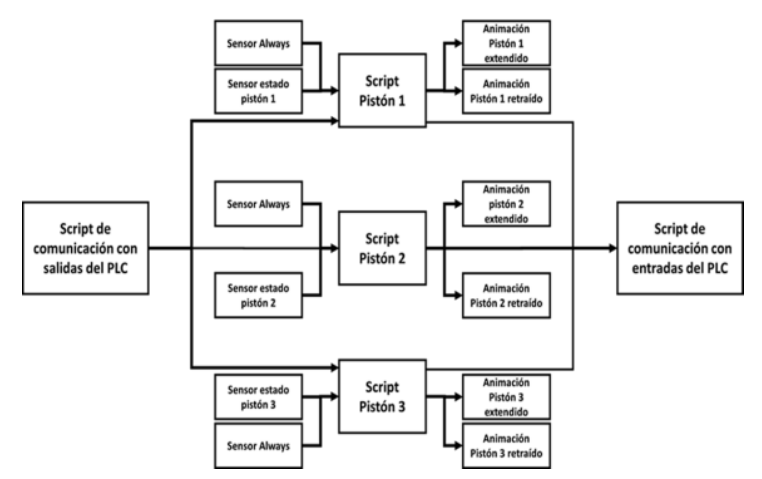

Figura 5 Diagrama de estados del escenario virtual Fuente: Elaboración Propia

A cada modelo digital de los pistones se le asignó un script desarrollado en lenguaje Python, cada script tiene como entradas; un sensor en modo "Always" (el cual permite correr de manera continua el script), un sensor de estado para saber la posición del pistón, y una conexión de entrada con el script general de comunicación hacia las variables de salidas del PLC.

CARMONA-MARTÍNEZ, Luis Alberto, GÓMEZ-HERNÁNDEZ, Alejandro, ORTÍZ-ORTÍZ, Tania Judith y GUALITO-OLVERA, Miguel Ángel. Desarrollo en Blender del gemelo digital de una estación electroneumática para su aplicación en laboratorios virtuales. Revista de Ingeniería Tecnológica. 2019 
En las salidas el script controla 2 actuadores de animación que permiten extender y retraer el pistón, y una conexión de salida con el script general de comunicación hacia las variables de entrada del PLC. Esta estructura permite controlar de manera externa e independiente cada uno de los pistones.

\section{Arquitectura de comunicación para equipos industriales}

Esta primera estación cuenta con una versatilidad en cuestión de comunicación, debido a la implementación de los protocolos industriales Modbus TCP y EtherNet/IP para los PLCs de Allen Bradley. Esto permite al estudiante utilizar e interactuar con distintos equipos de control que se comuniquen mediante los protocolos asignados. A continuación se da una breve descripción de los protocolos utilizados.

\section{Comunicación vía EtherNet/IP}

El protocolo EtherNet/IP es un empaquetado de mensajes a través del protocolo TCP/IP, estos mensajes utilizan el protocolo CIP al igual que los protocolos industriales ControlNet y DeviceNet. EtherNet/IP define su encapsulamiento a través del modelo OSI como se muestra en la Figura 6 (Prado, 2005).

\begin{tabular}{|c|l|}
\hline Aplicación & $\begin{array}{l}\text { Capa de aplicación CIP. } \\
\text { Biblioteca de objetos de aplicación. }\end{array}$ \\
\hline Presentación & $\begin{array}{l}\text { Servicios de gestión de datos CIP. } \\
\text { Mensajes explícitos, mensajes de E/S. }\end{array}$ \\
\hline Sesión & $\begin{array}{l}\text { CIP enrutamiento de mensajes, } \\
\text { gestión de conexión. }\end{array}$ \\
\hline Transporte & $\begin{array}{l}\text { Encapsulación CIP. } \\
\text { TCP/UDP. }\end{array}$ \\
\hline Red & IP. \\
\hline Enlace de & Ethernet. \\
\hline Fisica & Punto a punto, multicast, unicast. \\
\hline
\end{tabular}

Figura 6 Modelo de red EtherNet/IP Fuente: Elaboración Propia

La comunicación a través de CIP en EtherNet/IP utiliza dos métodos de comunicación (mensajes implícitos y mensajes explícitos), el método utilizado en este trabajo es el de mensajes explícitos, el cual se realiza a través de TCP/IP mediante el puerto 44818. Este método permite leer y escribir sobre una o varias variables en el PLC siguiendo la secuencia de comunicación mostrada en la figura 7.

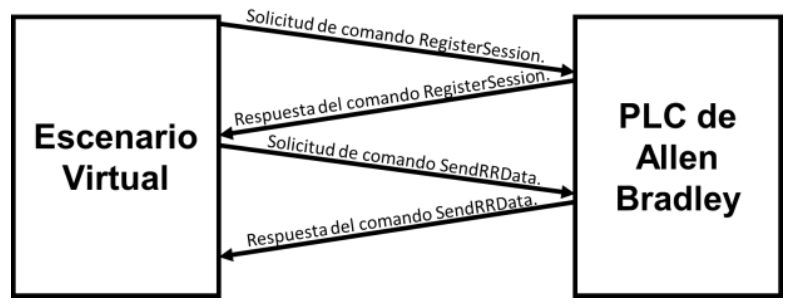

Figura 7 Diagrama de comunicación EtherNet/IP Fuente: Elaboración Propia

Bajo esta comunicación el PLC actúa como servidor y el escenario virtual como cliente, transfiriendo los estados de los sensores $\mathrm{y}$ actuadores en dos variables en el PLC. Con esta comunicación, se puede realizar conexión a través de Ethernet con los siguientes PLCs.
a) Micrologix
b) CompactLogix
c) ControlLogix
d) SoftLogix

Las pruebas de funcionamiento se realizaron mediante la utilización de un PLC CompactLogix L32E; programado con el software RSLogix 5000 (Figura 8) para el protocolo de comunicación EtherNet/IP.

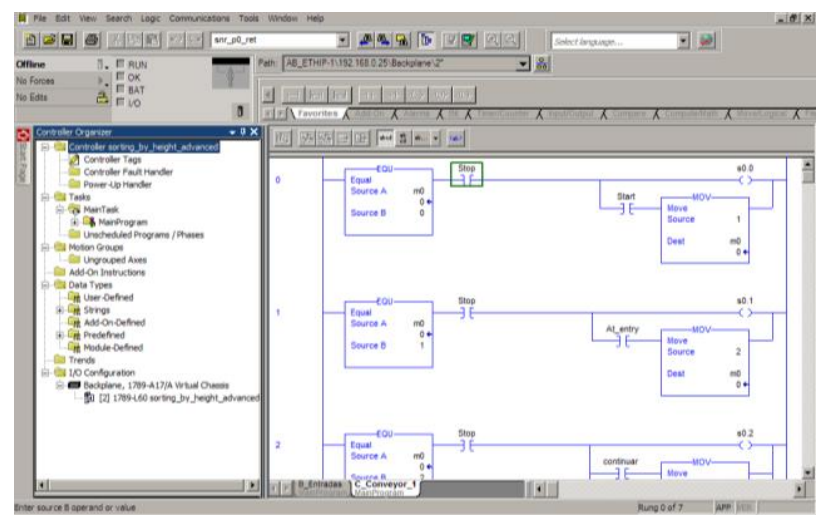

Figura 8 Programa PLC en RSLogix 5000

Fuente: Elaboración Propia

\section{Modbus TCP}

Modbus es un protocolo originalmente implementado para transferir datos a través de una capa serial, pero se ha expandido para su aplicación a través de TCP/IP utilizando la relación maestro-esclavo.

En esta comunicación el dispositivo maestro debe de iniciar una solicitud hacia un esclavo en específico y luego esperar una respuesta de ese esclavo, como se muestra en la figura 9 . 


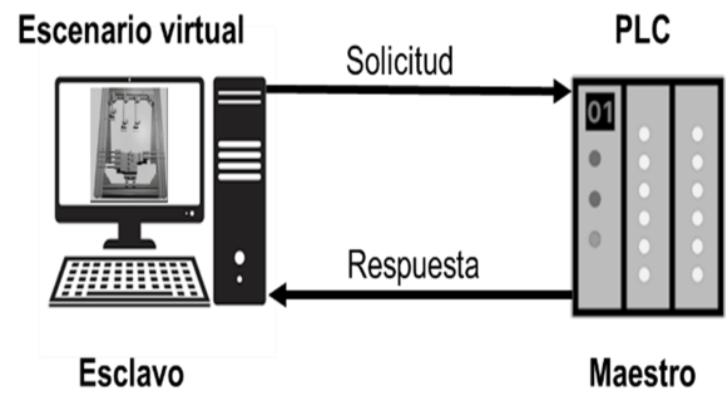

Figura 9 Diagrama de comunicación Modbus TCP Fuente: Elaboración Propia

Con el uso de la comunicación Modbus TCP este escenario virtual puede comunicarse con PLCs que empleen el mismo protocolo de comunicación, transfiriendo los estados de los sensores y actuadores a través del puesto TCP 502.

Las pruebas de funcionamiento se realizaron mediante la utilización de una Raspberry Pi 3; programado con el software Codesys $^{\circledR}$ (Figura 10) para el protocolo de comunicación Modbus TCP.

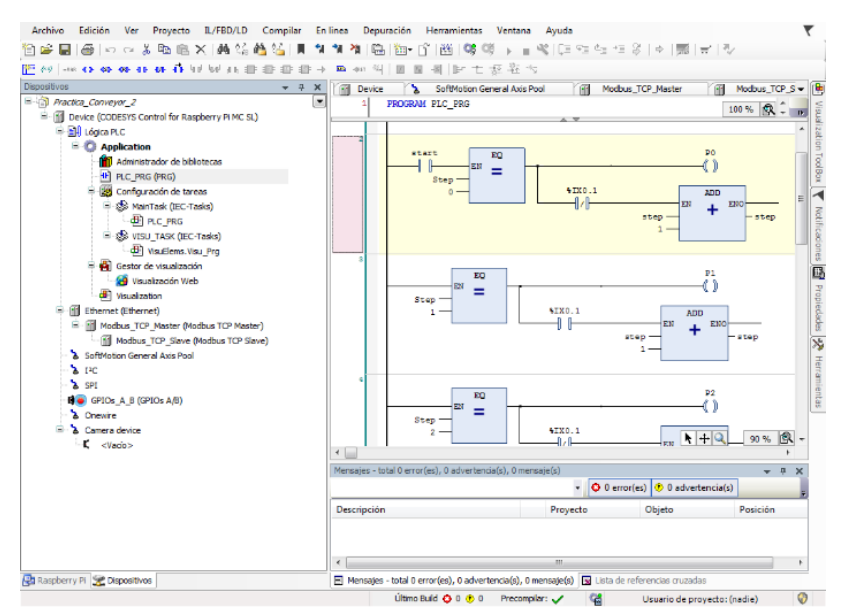

Figura 10 Programa Raspberry en Codesys Fuente: Elaboración Propia

\section{Impacto del laboratorio virtual}

El escenario desarrollado en este trabajo tiene un impacto directo en las siguientes carreras y materias dentro de la universidad tecnológica de Querétaro:

\section{Carrera en Mecatrónica:}

- Controladores lógicos programables.

- Microcontroladores.

- $\quad$ Fundamentos de instrumentación virtual.

- $\quad$ Integración de sistemas automáticos.
Carrera en tecnologías de la automatización.

- $\quad$ Instrumentación virtual.

- $\quad$ Sistemas de manufactura flexible.

- Control lógico avanzado

\section{Conclusiones}

Las conclusiones obtenidas de este proyecto son las siguientes:

- El desarrollo de este proyecto permite contar con una alternativa de bajo costo para la implementación de laboratorios virtuales en automatización, dentro de universidades y centros de capacitación.

- Este trabajo tiene contemplado implementar más escenarios para enriquecer el laboratorio virtual.

- $\quad$ Este trabajo es de gran impacto para las ingenierías; debido al crecimiento en automatización industrial, tanto en México como a nivel mundial.

\section{Agradecimientos}

Se agradece al cuerpo académico de automatización y control de la universidad tecnológica de Querétaro por el apoyo brindado para la aplicación de la metodología.

\section{Referencias}

Market Research Future. (2018). Industrial Automation Market Research Report- Forecast 2022. Obtenido de https://www.marketresearchfuture.com/reports/i ndustrial-automation-market-2212

Forbes México. (2019). Falta de automatización pone en riesgo la competitividad de México. Obtenido de https://www.forbes.com.mx/faltade-automatizacion-pone-en-riesgo-lacompetitividad-de-mexico/

Ortega-Moody, J., Sánchez-Alonso, R., Grise, W., García-Malacara, J., Vidana-Morales, R., Reyes-Morales, G. (2017). Laboratorio virtual de escenarios industriales para entrenamiento en las áreas de automatización y control. Pistas Educativas, 92(3), 285-287. DOI: http://dx.doi.org/10.6036/8051 
Lorandi, A., Hermida, G., Hernández, J. y Ladrón de Guevara, E. (2011). Los laboratorios virtuales y laboratorios remotos en la enseñanza de la ingeniería. Revista Internacional de Educación en Ingeniería, vol. 4, 24-30.

Márquez, D. y Cárdenas, A., (2008). Implementación de un Laboratorio Virtual para la enseñanza de Controladores PID. Revista Información Tecnológica, vol. 19, 75-78.

Hardware Meets Software. (2018). HMS Release 2016 Industrial Market Share Report. LC Automation. Obtenido de https://www.lcautomation.com/Page/Latest_Ne ws/2016_Industrial_Network_Market_Share_R eport.aspx

Prado Hernández, D. (2005). Desarrollo de un servidor de comunicaciones para el monitoreo, supervisión y control de equipos industriales. (Tesis inédita de ingeniería). Universidad Simón Bolívar, Sartenejas, Venezuela. 